\title{
Proton-Proton Total Cross-Section Based On New Data of Colliders and Cosmic Rays
}

\author{
Jorge Pérez-Peraza*, Alejandro Sánchez Hertz \\ Instituto de Geofísica, Universidad Nacional Autónoma de México, Coyoacán, México
}

Email adress:

perperaz@geofisica.unam.mx (J. Pérez-Peraza)

To cite this article:

Jorge Pérez-Peraza, Alejandro Sánchez Hertz. Proton-Proton Total Cross-Section Based On New Data of Colliders and Cosmic Rays. International Journal of High Energy Physics. Vol. 2, No. 2, 2015, pp. 27-33. doi: 10.11648/j.ijhep.20150202.12

\begin{abstract}
High energy colliders (accelerators) are fundamental tools in many branches of science. Similarly, cosmic rays observatories are one of the windows to study the universe and high energy particle processes. The last advances in these fields are respectively the LHC (Large Hadron Collider) and the Pierre Auger Observatory. Among the main subjects studied in hadronic physics is the proton-proton ( $p p$ ) elastic scattering. The Total Cross-Section $\left(\sigma_{\mathrm{pp}}\right)$, has been recently measured at 7 and 8 $\mathrm{TeV}$ in the LHC, and at $57 \mathrm{TeV}$ in the Pierre Auger Observatory. Importance of the $\sigma_{\mathrm{pp}}$ lies in studies of elastic and diffractive scattering of protons, and to model the development of showers induced by the interaction of ultra high energy cosmic rays in the atmosphere. The gap in data between accelerators and cosmic ray experiment energies does not allow for the exact knowledge of $\sigma_{\mathrm{pp}}$ with energy. Furthermore, since cosmic rays results are of indirect nature, there is consequently a high dispersion in predictions of different authors at this regard. Using the new data, we show here that within the frame of the first-order Glauber multiple diffraction theory the overall data fits very successfully. Our results shows that $\sigma_{\mathrm{pp}}$ grows more slowly (compared with previous predictions), within narrow error bands that avoid any fast slope change. We predict that the future experimental value at $13 \mathrm{TeV}$ from the LHC will fall nicely within our fitting curve. Our phenomenological approach allows for the calculation of $\sigma_{\mathrm{pp}}$ for any other energy value either at the colliders or cosmic ray energies. A deep knowledge, control and handle of hadron-hadron interactions at very high energies will have useful implications in many branches of physics.
\end{abstract}

Keywords: Cosmic Rays, Elastic Scattering, Hadronic Interactions

\section{Introduction}

An important process in the hadron physics is the $p p$ elastic scattering. In spite of the amount of currently available data and descriptive models of these data, actually there is not a satisfactory description based on pure Quantum Chromodynamics (QCD), that would be widely accepted in considering this dynamic process. The QCD perturbative theory cannot be extended to the weak interactions region and the QCD non-perturbative theory is not able to predict dispersion states. There are approaches based on QCD that try to explain the phenomenon which have been successful in describing processes where there is much transfer momentum, where quarks, which are the particles that compose hadrons behave as if they were free particles. In this case, the perturbative approach can be applied. On the other hand, in the region with low transfer momentum (the namely region of soft collisions), the effective coupling constant of strong interactions is large and therefore, perturbative approach cannot be applied. Historically, the study of the total cross section, which measure the total interaction probability has played a fundamental role in nuclear and particle physics. For energies of only a few $\mathrm{GeV}$, the total cross section in hadrons scattering usually has a complicated structure composed with peaks or resonances, which reveals the formation of excited hadronic states. On the other side, for higher energies, the total cross section have a softer behaviour.

Also it has been extensively investigated the $p p$ elastic dispersion, mainly in the region where there is a small transfer momentum, which is where there is a great number of experimental data available, although in some specific energies data have been obtained in the region where there is large transfer momentum. An important feature that have resulted of the analysis of experimental data, is the discovery that the effective range of interaction in hadron collision increases in accordance with the energy growth. In the same way, it has been discovered that the probability of absorption also increases; namely, that particles appear to expand and become blacker for high energies. 
In this work we develop a prediction based on a purely diffractive model that approximates reasonably the existing experimental data of particle accelerators and cosmic ray observatories for $p p$ and $\bar{p} p$ total cross section in the Center of Mass Energy range: $10-10^{5} \mathrm{GeV}$, including the values of 7 and $8 \mathrm{TeV}$ obtained in the LHC at the CERN in 2011 and 2013 [1-4]. We also consider here the new value at $57 \mathrm{TeV}$ from the Pierre Auger Observatory obtained in July 2012 [5].

\section{Elastic Hadronic Scattering Amplitude}

To know how much the hadrons disperse during an elastic collision, an hadronic scattering amplitude must be constructed. One way to construct the hadronic amplitude from the most elemental frame is by means of Glauber's multiple diffraction theory. The approach is based on the impact parameter and eikonal formalisms as follows: assuming azimuthal symmetry in the collision of two hadrons (neglecting the spin), in our case two protons, we have the following expression for the elastic hadronic scattering amplitude:

$$
F(q, s)=\int_{0}^{\infty}\left[1-e^{-\Omega(b, s)}\right] J_{0}(q b) b d b .
$$

here $q^{2}=-t$ is the four-momentum transfer squared, $\sqrt{s}$ is the center of mass energy, $b$ the impact parameter, $J_{0}$ the zero- ${ }^{-}$order Bessel and $\Omega$ is a function of real values which is used to describe the opacity of hadrons. The equation describing $\Omega$ is the following:

$$
\Omega(\mathrm{b}, \mathrm{s})=4 \pi \int_{0}^{\infty} G^{2} \operatorname{Im} f(q, s) J_{0}(q b) q d q,
$$

where $G$ is the hadronic form factor:

$$
G=\left(1+\frac{q^{2}}{\alpha^{2}}\right)^{-1}\left(1+\frac{q^{2}}{\beta^{2}}\right)^{-1}
$$

and $\operatorname{Im} f(q, s)$ is the imaginary part of the elementary parton-parton amplitude:

$$
\operatorname{Im} f(q, s)=C \frac{1-\left(q^{2} / a^{2}\right)}{1+\left(q^{4} / a^{4}\right)},
$$

where $\alpha^{2}, \beta^{2}, a^{2}$ and $C$ are energy dependency parameters of real values. The proportionality factor $C$, is known as the "absorption factor".

\section{The $\rho$ Parameter}

The $\rho$ parameter is an experimental value that is obtained in particle accelerators, and is equal to the ratio of the real part to the imaginary part of the hadronic scattering amplitude $F$ at $q$ $=0$ :

$$
\rho(s)=\frac{\operatorname{Re} F(0, s)}{\operatorname{Im} F(0, s)} .
$$

On high energies this amplitude is mainly imaginary, but a knowledge of the real part allows us to obtain predictions of the total cross section (even for high energies) by means of scattering relations.
For obtain the fit of $\rho$ we have used the experimental data that have been obtained in particle accelerators in the Center of Mass (CM) energy range: $13.8-1800 \mathrm{GeV}$, that is, from the Alternating Gradient Synchrotron (AGS) to the Tevatron. With these experimental data we have calculated the fit for the whole range of energy: $10-10^{5} \mathrm{GeV}$, and thus, we have predicted reasonably the value obtained at $7 \mathrm{TeV}$ in the $\mathrm{LHC}$ of CERN. The form of the fit is the following:

$$
\rho(s)=\frac{A_{1} \ln \left(s / s_{0}\right)}{1+A_{2}\left[\ln \left(s / s_{0}\right)\right]+A_{3}\left[\ln \left(s / s_{0}\right)\right]^{2}},
$$

where $s_{0}=400 \mathrm{GeV}^{2}$ controls the point where $\rho$ reaches the zero and where the coefficients $A_{1}, A_{2}$ and $A_{3}$ control the maximum and the asymptotic behaviour, which values are the next:

$$
A_{1}=0.0702, A_{2}=0.3691, A_{3}=1.502 \times 10^{-3} .
$$

The results are shown in table 1 and figure 1 . As we can see, the $\rho$ parameter has negative values at $\mathrm{CM}$ energies less than approximately $21 \mathrm{GeV}$, and moreover, this present an asymptotic growth for energies greater than $4 \mathrm{TeV}$. In the same way we can see that the fit presents a reasonably approximation to the value obtained by the $\mathrm{LHC}$ at $7 \mathrm{TeV}$.

Table 1. Predicted and experimental values of $\rho$. In the last column are shown the references from which the experimental data were obtained: the Alternating Gradient Synchrotron (AGS), the Intersecting Storage Ring (ISR), the collaboration UA4/2 of the Super Proton Synchrotron (SPS), the collaboration E-710 of the Tevatron and the TOTEM collaboration of the Large Hadron Collider (LHC).

\begin{tabular}{llll}
\hline$\sqrt{\boldsymbol{s}}(\mathbf{G e V})$ & $\boldsymbol{\rho}$ (pred.) & $\boldsymbol{\rho}$ (exp.) & References \\
\hline 13.8 & -0.072 & $-0.074 \pm 0.018$ & AGS [6] \\
19.4 & -0.004 & $0.019 \pm 0.016$ & Fermilab [6] \\
23.5 & 0.020 & $0.020 \pm 0.050$ & ISR [7] \\
30.7 & 0.046 & $0.042 \pm 0.011$ & ISR [7] \\
44.7 & 0.071 & $0.062 \pm 0.011$ & ISR [7] \\
52.8 & 0.080 & $0.078 \pm 0.010$ & ISR [7] \\
62.5 & 0.086 & $0.095 \pm 0.011$ & ISR [7] \\
541 & 0.132 & $0.135 \pm 0.015$ & SPS UA4/2 [8] \\
1800 & 0.142 & $0.140 \pm 0.069$ & Tevatron E-710 [9] \\
7000 & 0.149 & $0.145 \pm 0.091$ & LHC TOTEM [3] \\
\hline
\end{tabular}

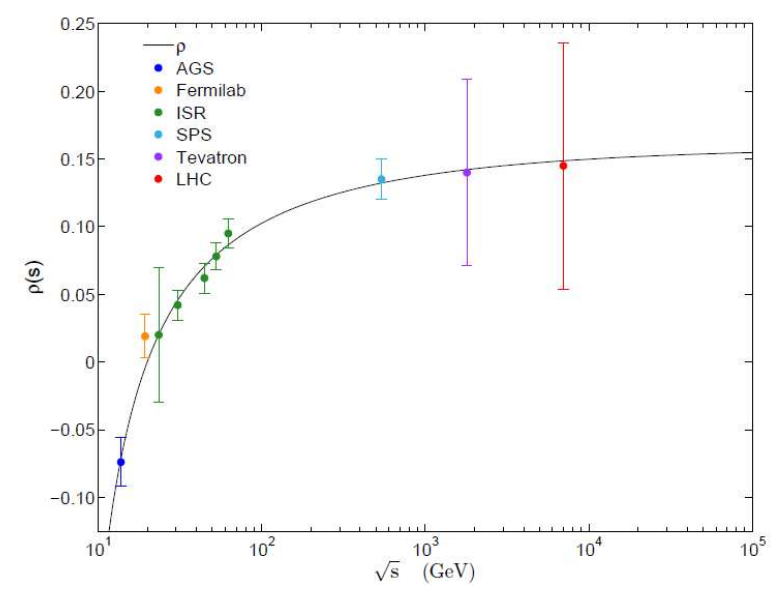

Figure 1. Calculated fit for the $\rho$ parameter (6), together with the experimental data from particle accelerators (see table 1). 


\section{Obtaining the Fits for the Energy Dependent Parameters}

With the values that were obtained for the energy dependent parameters (table 2), we have calculated the fits (parametrizations) for each one of those, which allow us to extrapolate our calculations to energies greater than 1800 $\mathrm{GeV}$.

Table 2. Calculated values for the energy dependent parameters: $C, \alpha^{-2}$ and $\lambda$. On the second column, $s_{0}=1$ GeV ${ }^{2}$ For the pp dispersion we have used the energies: 13.8 to $62.5 \mathrm{GeV}$, and for the $\bar{p} p$ dispersion: 546, 630 y $1800 \mathrm{GeV}$.

\begin{tabular}{|c|c|c|c|c|c|c|c|}
\hline$\sqrt{s}(\mathrm{GeV})$ & $\ln (s / s 0)$ & $\mathrm{C}(\mathrm{s})\left(\mathrm{GeV}^{-2}\right)$ & $\alpha^{-2}(s)\left(G e V^{-2}\right)$ & $\lambda(\mathbf{s})$ & $\mathrm{C} \boldsymbol{\alpha}^{2}$ & Dispersion Type & References \\
\hline 13.8 & 5.25 & 9.86 & 2.092 & -0.094 & 4.713 & $p p$ & Fermilab [10-13] \\
\hline 19.4 & 5.93 & 9.96 & 2.128 & 0.024 & 4.680 & $p p$ & Fermilab [10-13] \\
\hline 23.5 & 6.31 & 10.16 & 2.174 & 0.025 & 4.673 & $p p$ & ISR [14] \\
\hline 30.7 & 6.85 & 10.37 & 2.222 & 0.053 & 4.667 & $p p$ & ISR [14] \\
\hline 44.7 & 7.60 & 10.82 & 2.299 & 0.079 & 4.706 & $p p$ & ISR [14] \\
\hline 52.8 & 7.93 & 11.12 & 2.350 & 0.099 & 4.732 & $p p$ & ISR [14] \\
\hline 62.5 & 8.27 & 11.42 & 2.400 & 0.121 & 4.758 & $p p$ & ISR [14] \\
\hline 546 & 12.60 & 17.44 & 2.915 & 0.180 & 5.983 & $p p$ & SPS UA4 $[15,16]$ \\
\hline 630 & 12.89 & 17.80 & 2.948 & 0.184 & 6.038 & $\bar{p} p$ & SPS UA4 [17] \\
\hline 1800 & 14.99 & 22.41 & 3.310 & 0.199 & 6.770 & $\bar{p} p$ & Tevatron E-710 [18] \\
\hline
\end{tabular}

Based on the behavior that is shown by both $C$ and $\alpha^{-2}$ parameters, we can see that their data sets are statistically consistent with quadratic polynomials for $[\ln (s)]^{2}$, so that using linear regression we have obtained the following fits for each one of them:

$$
\begin{gathered}
C(s)=11.97-1.003 \ln \left(s / s_{0}\right)+0.1134\left[\ln \left(s / s_{0}\right)\right]^{2} \mathrm{GeV}^{-2}, \\
\alpha^{-2}(s)=1.8+2.8 \times 10^{-2} \ln \left(s / s_{0}\right)+4.8 \times 10^{-3}\left[\ln \left(s / s_{0}\right)\right]^{2} \mathrm{GeV}^{-2},
\end{gathered}
$$

where we have taken $s_{0}=1 \mathrm{GeV}^{2}$. In figures 2 and 3 we show the previous fits together with the corresponding values of table 2. As we can see, both present positive curves with the energy increase. The dimensionless product $C \alpha^{2}$ provides us with information of the blackening and expansion in elastic hadron scattering, the plot of this product can be seen in figure 4 .

Since we know that the parameter $\lambda$ has a very similar behaviour of the $\rho$ parameter, we have used a similar fit:

$$
\lambda(s)=\frac{A_{1} \ln \left(s / s_{0}\right)}{1+A_{2} \ln \left(s / s_{0}\right)+A_{3}\left[\ln \left(s / s_{0}\right)\right]^{2}},
$$

where $s_{0}=400 \mathrm{GeV}^{2}$ controls the point where $\lambda$ reaches zero, and the coefficients $A_{1}, A_{2}$ and $A_{3}$ control the maximum and the asymptotic behaviour of the fit, whose values are:

$$
A_{1}=0.088, A_{2}=0.334, A_{3}=2.737 \times 10^{-7} \text {. }
$$

In figure 5 we can see the obtained fit for $\lambda$.

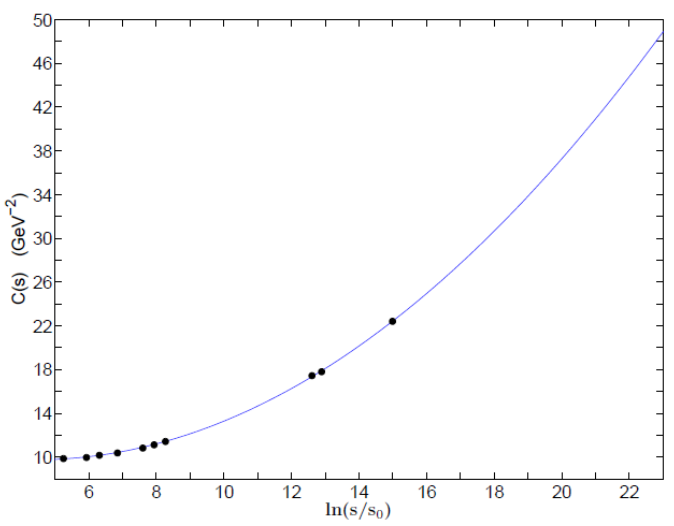

Figure 2. Obtained fit for the C parameter (8). The dots are the data of table 2.

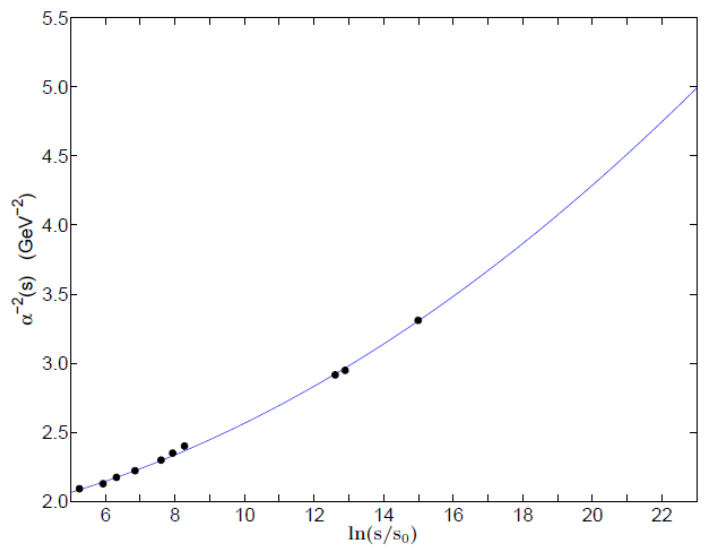

Figure 3. Obtained fit for the $\alpha^{-2}$ parameter (9). The dots are the data of table 2 .

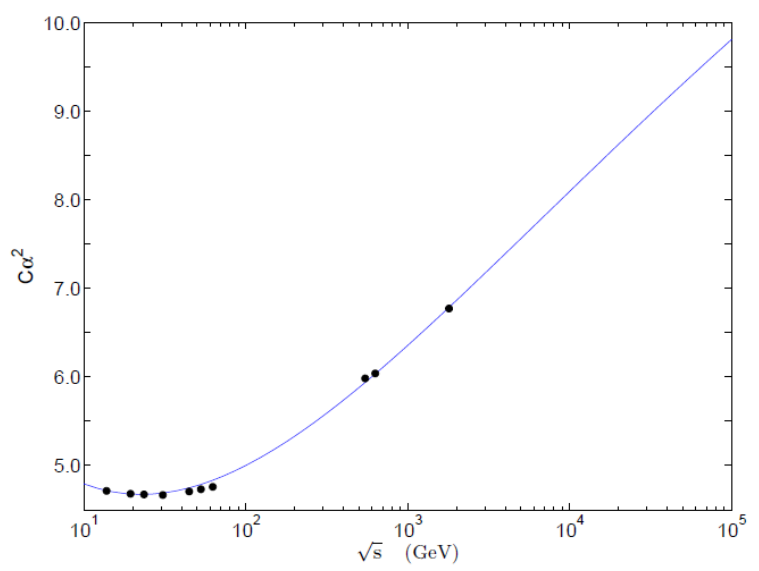

Figure 4. Predictions for the dimensionless quantity $\mathrm{Ca}^{2}$. The dots are the data of table 2. 


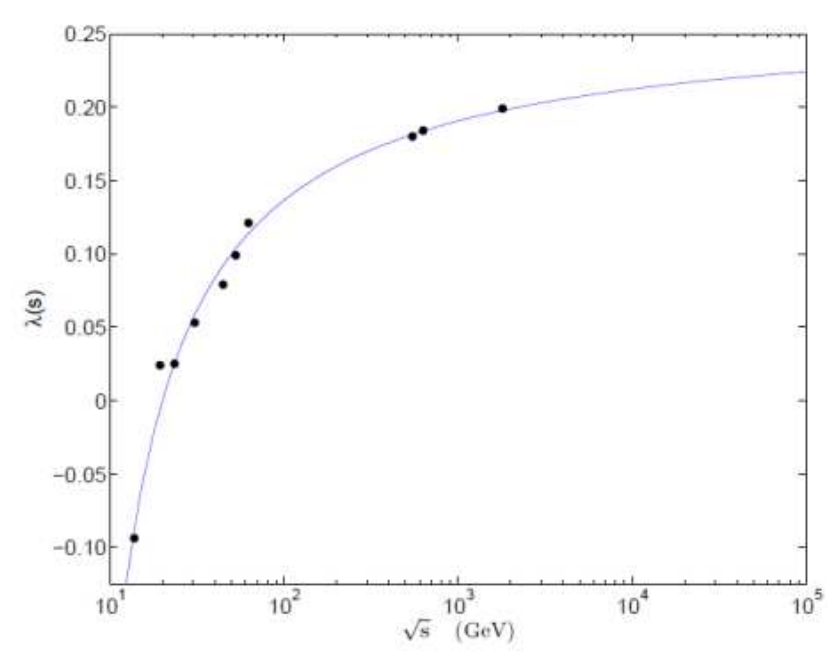

Figure 5. Predictions for the $\lambda$ parameter $\lambda$ (10). The dots are the data of table 2 .

\section{Derivation of the $p p$ Total Cross Section}

In the absence of a QCD description for this phenomenon, a number of models and phenomenological approximations have been developed to describe the available data. Though these formalisms do not give a final answer to the basic involved processes, they are however useful tools that allow for geometric and dynamic assumptions, that lead to reproduce the experimental data. Geometrical models based on the Multiple Diffraction theory of Glauber [19, 20] have proved to be good phenomenological approaches. An essential feature in the multiple diffraction formalism is the connection of the elastic dispersion cross-sections for composite particles (originally for nuclei and after for nucleons) with the dispersion amplitudes of their individual components.

Following this theory, we present here a prediction of the $p p$ dispersion based on an eikonal (a symmetrical two-dimensional Fourier transform) that depends on parameters describing the hadronic form factor and the elementary parton-parton amplitude. By means of this eikonal, we then calculate the real and imaginary parts of the hadronic scattering amplitude. With this amplitude and with the fits for the parameters associated with the eikonal, we have obtained a prediction curve for the $p p$ total cross section.

For the calculation of the total cross-section $\sigma_{p p}$ we have first obtained the differential cross section $(\mathrm{d} \sigma / \mathrm{dt})$ for each one of the experimental data of the accelerators whose operation were previous to the LHC: which corresponds to the energies range $13.8-1800 \mathrm{GeV}$. The fit for $\mathrm{d} \sigma / \mathrm{dt}$ have been obtained by means of the real and imaginary part of the hadronic scattering amplitude $F$ (equation (1)), which is dependent of the energy and transfer momentum, such as is described by the following equation:

$$
\frac{d \sigma}{d t}=\pi\left[\operatorname{Re} F(q, s)^{2}+\operatorname{Im} F(q, s)^{2}\right]
$$

Figure 6 shows an example for the energy of $52.8 \mathrm{GeV}$, where we can see a reasonably concordance with the experimental data. Figures for the remaining energies can be consulted in [21].

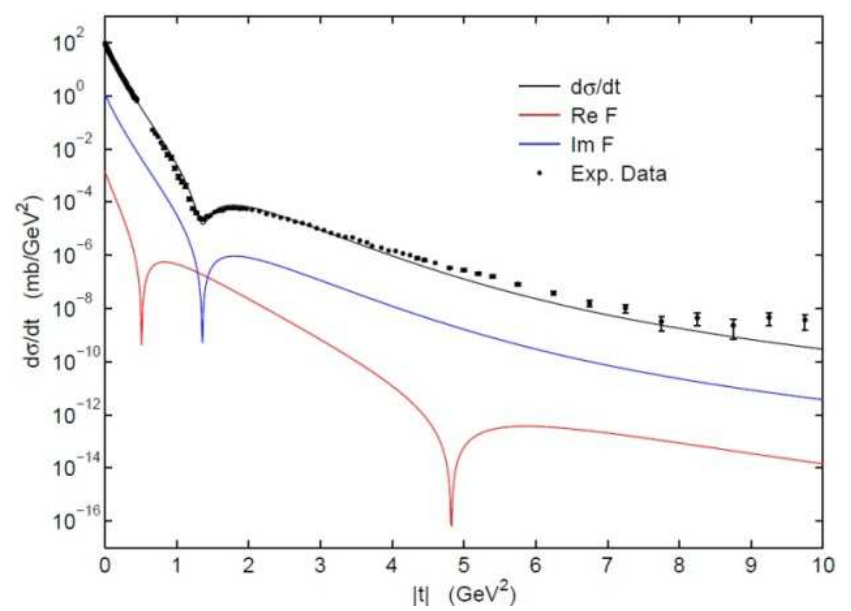

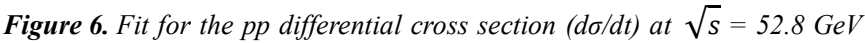
compared with the experimental data.

The knowledge of $\mathrm{d} \sigma / \mathrm{dt}$ is also very important for the study of $p p$ elastic dispersion.

With the fits for the energy dependent parameters: $C, \alpha^{-2}$ and $\lambda$, we have thus completely determined all the parameters associated with the eikonal, and then, we can now to calculate the $p p$ total cross section by means of the following expression:

$$
\sigma_{p p}=4 \pi \int_{0}^{\infty}\left\{1-e^{-\Omega(b, s)} \cos [\lambda \Omega(b, s)]\right\} b d b
$$

where the integrand represents the imaginary part of the elastic hadronic scattering amplitude and $\lambda$ is, as we mentioned before, an energy dependent parameter describing the proportionality between the real and imaginary parts. The procedure to derive the previous equation is somewhat complex and was extensively described in [21-24].

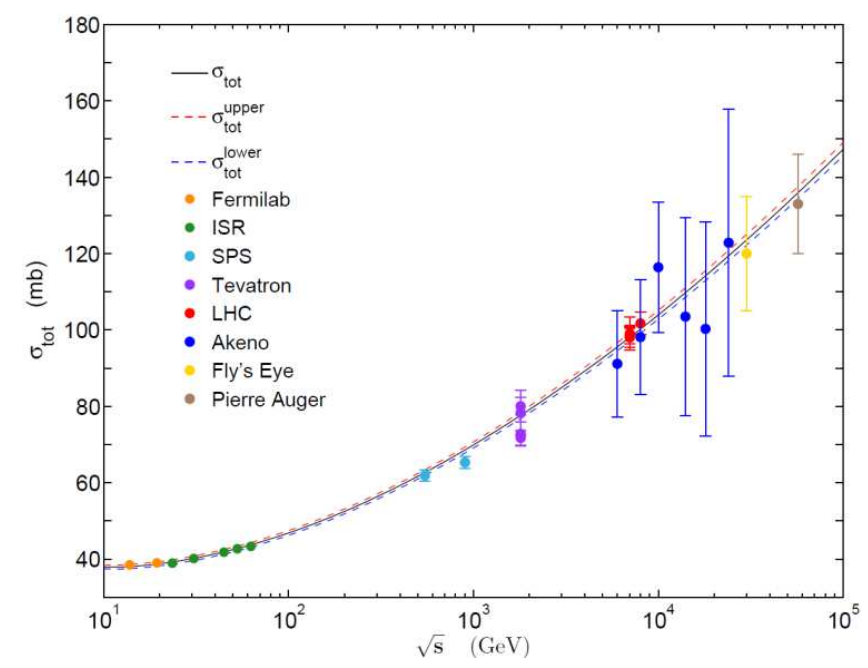

Figure 7. Fitting of the pp total cross section with the predicted error bands in the energy interval: $10-10^{5} \mathrm{GeV}$. Also are shown the experimental data of particle accelerators and cosmic rays observatories (see tables 3 and 4).

In figure 7 we show the result obtained for the $p p$ total 
cross section, calculated by means of (13) and the fits for the energy dependent parameters (8-11), in the energies range: 10 to $10^{5} \mathrm{GeV}$. We have calculated Error Bands with $95 \%$ of prediction for each of the involved parameters. Further, we know that the significance $\delta$ is related with the prediction percentage by means of: $100(1-\delta) \%=95 \%$. Therefore, we have that $\delta=0.05$ and the Student's $t$ that we require is $t_{\delta / 2}=t_{0.025}$ for $\mathrm{n}-2=8$ degrees of freedom, which is equal to 2.306 .

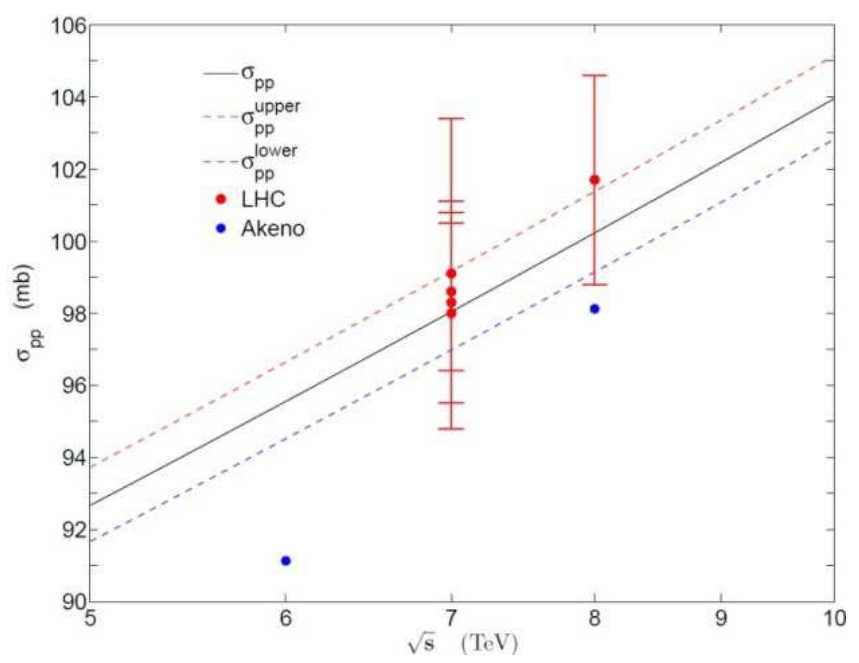

Figure 8. Close Up of the fitting of the pp total cross section with the predicted error bands in the energy interval: $5-10 \mathrm{TeV}$. Also are shown the experimental data obtained by the LHC and Akeno (see tables 3 and 4).

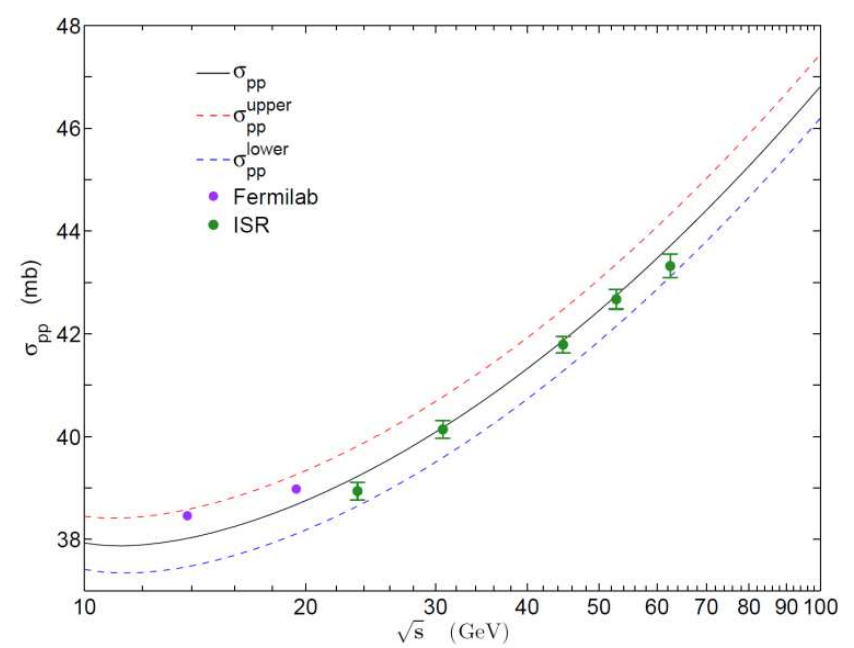

Figure 9. Close Up of the fitting of the pp total cross section with the predicted error bands in the energy interval: 10-100 GeV. Also are shown the experimental data obtained by the Fermilab and ISR (see tables 3 and 4).

Fig. 7 also shows the experimental data that have been obtained in particle accelerators and cosmic ray observatories (see table 3 ). With the aim of better resolution in figures 8 and 9 we can see the same results, but for two different energy intervals. As we can see from the previous figures and table 3 , the fit that we have calculated for the $p p$ total cross section reasonably agrees with the particle accelerators data: from that obtained by the Fermilab to the LHC. With respect to the results of the cosmic rays observatories, our prediction is quite consistent with the
Akeno's data, the value of Fly's Eye, and the result of Pierre Augier Observatory.

In table 4 are shown the calculated values for the upper and lower prediction bands of energies in which there exist experimental data. In the fifth and sixth columns we can see the absolute differences that there is between each one of the prediction bands with respect to the central prediction, that is, $\Delta \sigma_{1}=\left|\sigma_{p p}-\sigma_{p p}^{\text {upper }}\right|$ and $\Delta \sigma_{2}=\left|\sigma_{p p}-\sigma_{p p}^{\text {lower }}\right|$. We can observe that for energies less or equal than $1800 \mathrm{GeV}$ the absolute differences are practically the same, that is, we can say that we have an \pm ; while, for energies greater than $1800 \mathrm{GeV}$, the absolute differences between the upper and lower prediction bands begins to slightly increment. This is mainly due to the fact that the dispersion in the measurements is not uniform for each one of the energy dependent parameters. On the other hand, we can also see that the error bands begin to get wider for energies greater than $1800 \mathrm{GeV}$. This occurs because from this energy is where the extrapolation of the energy dependent fits begins, and therefore, the uncertainty in the prediction tends to increase for higher energies.

It should be mentioned that for the energy values (546, 630 and $1800 \mathrm{GeV}$ ) we used data of $\bar{p} p$ dispersion, because there are not $p p$ experimental data for higher energies than the ISR energies and less than the LHC energies, namely, for $62.5 \mathrm{GeV}<\sqrt{s}<7 \mathrm{TeV}$. However, from the analysis of the existing experimental data at present, for both reactions, it is known that for higher energies than approximately 35 $\mathrm{GeV}$, the $\bar{p} p$ and $p p$ total cross sections tend to be equal [25], which justifies our calculations.

\section{Comparison with a Previous Fit}

In a previous work [24]. a fit was obtained using the same methodology that we have employed in the present work, except that the fits for the energy dependent parameters were different, since these were made using a distinct approach for the experimental data of the differential cross sections. In figure 10 are shown both fits, together with the experimental data. The black line corresponds to the prediction of this work and the red line to that published in [24]. In table 5 we can see the obtained values for both fits at energies in which there is experimental data. In the fourth column is shown the absolute difference between both predictions. As we can see, this difference begins to increase from energies greater than $18 \mathrm{TeV}$. For the energy of $57 \mathrm{TeV}$, where the Pierre Augier Observatory has presented a value of $133 \mathrm{mb}$, we can see that in the present work we have obtained a value of 135.93 $\mathrm{mb}$, while in [24] it was obtained a value of $139.31 \mathrm{mb}$, so, that with the fit of the present work we are more close to the result of the Pierre Augier experiment. For the energies of 7 and $8 \mathrm{TeV}$, where the LHC present its results, both fits agree reasonably. In the same way, a good match are obtained for the energy range: $10-200 \mathrm{GeV}$, that is the energy region that corresponds to the Fermilab and ISR experimental data. 


\section{Conclusions}

Our results adequately describe the experimental data obtained in particle accelerators and cosmic rays observatories for the energy range: $10-10^{5} \mathrm{GeV}$, including the most recent values of 7 and $8 \mathrm{TeV}$ published by the LHC (CERN) and the value presented by the Observatory Pierre Auger at $57 \mathrm{TeV}$. Our results have also improved the previous approaches, published a few years before the current data of the LHC and the Pierre Auger Observatory were found: especially those in [24], whose fits showed a good prediction for the data in the range of energies: $1-100 \mathrm{TeV}$, few years before the current data of the LHC and the Pierre Auger Observatory were found. Due to the fact that at present our results are the most consistent with experimental data, both from accelerators and cosmic ray observatories, we expect that the same fitting trend will continue when new high energy data values will be available. At the moment we predict the next experimental value at $13 \mathrm{TeV}$ (at CERN) to be $\sigma_{p p}=108.44_{-107.27}^{+109.7} \mathrm{mb}$, which falls nicely within our fitting curve. Our model allows now to predict any value of $\sigma_{p p}$ at any other energy.

Table 3. Results for the pp total cross section. Values shown in the second column correspond to the calculated by means of (13). Values shown in the third column correspond to the experimental data obtained in particle accelerators and cosmic ray observatories. The reference of these can be seen in the last column.

\begin{tabular}{|c|c|c|c|}
\hline$\sqrt{s}(\mathrm{GeV})$ & $\begin{array}{l}\sigma_{p p} \text { (pred.) } \\
(\mathrm{mb})\end{array}$ & $\sigma_{p p}(\exp ).(\mathrm{mb})$ & References \\
\hline 13.8 & 38.02 & $38.46 \pm 0.04$ & Fermilab $[25,26]$ \\
\hline 19.4 & 38.68 & $38.98 \pm 0.04$ & Fermilab $[25,26]$ \\
\hline 23.5 & 39.22 & $38.94 \pm 0.17$ & ISR [27] \\
\hline 30.7 & 40.18 & $40.14 \pm 0.17$ & ISR [27] \\
\hline 44.7 & 41.87 & $41.79 \pm 0.16$ & ISR [27] \\
\hline 52.8 & 42.74 & $42.67 \pm 0.19$ & ISR [27] \\
\hline 62.5 & 43.71 & $43.32 \pm 0.23$ & ISR [27] \\
\hline \multirow[t]{2}{*}{546} & 62.64 & $61.26 \pm 0.93$ & Tevatron CDF [28] \\
\hline & & $61.9 \pm 1.5$ & SPS UA4 [29] \\
\hline 900 & 68.56 & $65.3 \pm 1.5$ & SPS UA5 [30] \\
\hline \multirow[t]{4}{*}{1800} & 77.62 & $71.71 \pm 2.02$ & Tevatron E-811 [31] \\
\hline & & $72.8 \pm 3.1$ & Tevatron E-710 [32] \\
\hline & & $78.3 \pm 5.9$ & Tevatron E-710 [33] \\
\hline & & $80.03 \pm 2.24$ & Tevatron CDF [29] \\
\hline 6000 & 95.55 & $91.13 \pm 14$ & Akeno [34] \\
\hline \multirow[t]{4}{*}{7000} & 98.04 & $98.3 \pm 2.8$ & LHC TOTEM [1] \\
\hline & & $98.6 \pm 2.2$ & LHC TOTEM [2] \\
\hline & & $98.0 \pm 2.5$ & LHC TOTEM [3] \\
\hline & & $99.1 \pm 4.3$ & LHC TOTEM [3] \\
\hline \multirow[t]{2}{*}{8000} & 100.23 & $98.12 \pm 15$ & Akeno [35] \\
\hline & & $101.7 \pm 2.9$ & LHC TOTEM [4] \\
\hline 10000 & 103.95 & $116.4 \pm 17$ & Akeno [35] \\
\hline 14000 & 109.74 & $103.49 \pm 26$ & Akeno [35] \\
\hline 18000 & 114.18 & $100.27 \pm 28$ & Akeno [35] \\
\hline 24000 & 119.40 & $122.85 \pm 35$ & Akeno [35] \\
\hline 30000 & 123.54 & $120 \pm 15$ & Fly’s Eye [36] \\
\hline 57000 & 135.93 & $133 \pm 13$ & Pierre Auger [5] \\
\hline
\end{tabular}

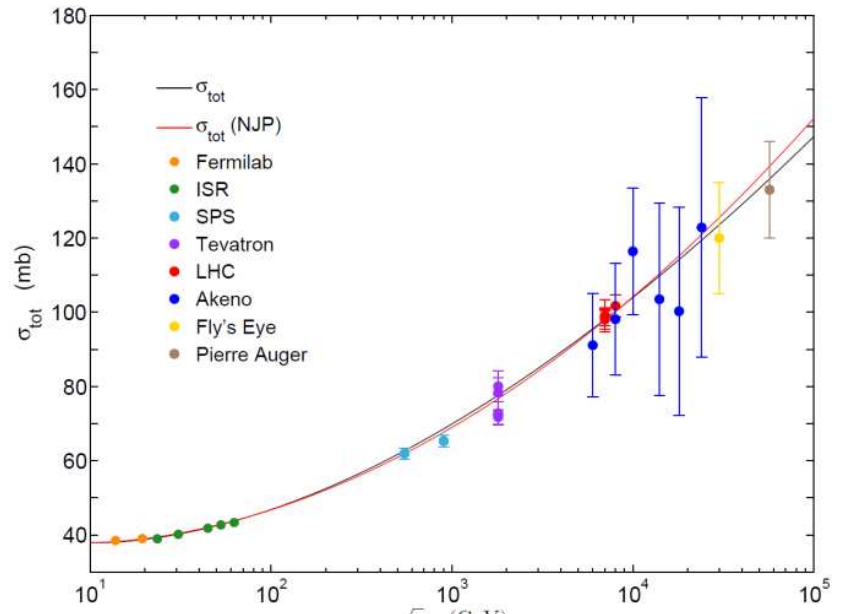

Figure 10. Fittings for the pp total cross section. Black line corresponds to the fit of the present work and red line to the fit published in the NJP. Also are shown the experimental data (see tables 3 and 5).

Table 4. Calculated prediction bands for the pp total cross section. In the third column are shown the obtained values for the upper prediction band and in the fourth column the values for the lower prediction band. In the fifth and sixth columns are shown the absolute difference that exists between both bands with respect to the central fit.

\begin{tabular}{|c|c|c|c|c|c|}
\hline $\begin{array}{l}\sqrt{s} \\
(\mathrm{GeV})\end{array}$ & $\begin{array}{l}\sigma_{p p} \\
(\mathbf{m b})\end{array}$ & $\begin{array}{l}\sigma_{p p}^{\text {upper }} \\
(\mathrm{mb})\end{array}$ & $\begin{array}{l}\sigma_{p p}^{\text {lower }} \\
(\mathrm{mb})\end{array}$ & $\Delta \sigma_{1}(\mathrm{mb})$ & $\Delta \sigma_{2}(\mathrm{mb})$ \\
\hline 13.8 & 38.02 & 38.58 & 37.47 & 0.56 & 0.55 \\
\hline 19.4 & 38.68 & 39.26 & 38.11 & 0.58 & 0.57 \\
\hline 23.5 & 39.22 & 39.81 & 38.65 & 0.59 & 0.57 \\
\hline 30.7 & 40.18 & 40.77 & 39.59 & 0.59 & 0.59 \\
\hline 44.7 & 41.87 & 42.47 & 41.27 & 0.60 & 0.60 \\
\hline 52.8 & 42.74 & 43.35 & 42.15 & 0.61 & 0.59 \\
\hline 62.5 & 43.71 & 44.33 & 43.11 & 0.62 & 0.60 \\
\hline 546 & 62.64 & 63.39 & 61.93 & 0.75 & 0.71 \\
\hline 900 & 68.56 & 69.36 & 67.80 & 0.80 & 0.76 \\
\hline 1800 & 77.62 & 78.51 & 76.77 & 0.89 & 0.85 \\
\hline 6000 & 95.55 & 96.64 & 94.52 & 1.09 & 1.03 \\
\hline 7000 & 98.04 & 99.16 & 96.98 & 1.12 & 1.06 \\
\hline 8000 & 100.23 & 101.38 & 99.14 & 1.15 & 1.09 \\
\hline 10000 & 103.95 & 105.15 & 102.83 & 1.20 & 1.12 \\
\hline 14000 & 109.74 & 111.01 & 108.54 & 1.27 & 1.20 \\
\hline 18000 & 114.18 & 115.51 & 112.93 & 1.33 & 1.25 \\
\hline 24000 & 119.40 & 120.80 & 118.09 & 1.40 & 1.31 \\
\hline 30000 & 123.54 & 125.01 & 122.18 & 1.47 & 1.36 \\
\hline 40000 & 129.01 & 130.55 & 127.58 & 1.54 & 1.43 \\
\hline 57000 & 135.93 & 137.57 & 134.41 & 1.64 & 1.52 \\
\hline
\end{tabular}


Table 5. Some values of the pp total cross section fittings. In the second column are shown the values obtained in the present work and in third column the values published in NJP [6]. In the fourth column it can be see the absolute difference between both.

\begin{tabular}{|c|c|c|c|}
\hline $\begin{array}{l}\sqrt{s} \\
(\mathrm{GeV})\end{array}$ & $\begin{array}{l}\sigma_{p p}(\text { present } \\
\text { work) }(\mathrm{mb})\end{array}$ & $\begin{array}{l}\sigma_{p p}(\mathrm{NJP}) \\
(\mathrm{mb})\end{array}$ & Absolute difference (mb) \\
\hline 13.8 & 38.02 & 38.18 & 0.16 \\
\hline 19.4 & 38.68 & 38.90 & 0.22 \\
\hline 23.5 & 39.22 & 39.46 & 0.24 \\
\hline 30.7 & 40.18 & 40.41 & 0.23 \\
\hline 44.7 & 41.87 & 42.05 & 0.18 \\
\hline 52.8 & 42.74 & 42.89 & 0.15 \\
\hline 62.5 & 43.71 & 43.81 & 0.10 \\
\hline 546 & 62.64 & 61.86 & 0.78 \\
\hline 900 & 68.56 & 67.66 & 0.90 \\
\hline 1800 & 77.62 & 76.60 & 0.92 \\
\hline 6000 & 95.55 & 95.21 & 0.34 \\
\hline 7000 & 98.04 & 97.83 & 0.21 \\
\hline 8000 & 100.23 & 100.15 & 0.08 \\
\hline 10000 & 103.95 & 104.13 & 0.18 \\
\hline 14000 & 109.74 & 110.35 & 0.61 \\
\hline 18000 & 114.18 & 115.18 & 1.00 \\
\hline 24000 & 119.40 & 120.90 & 1.50 \\
\hline 30000 & 123.54 & 125.47 & 1.93 \\
\hline 57000 & 135.93 & 139.31 & 3.38 \\
\hline
\end{tabular}

\section{Acknowledgements}

We thank the UNAM for supporting this research by means of the grant IN106214 DGAPA-PAPIIT.

\section{References}

[1] G. Antchev, et al., EPL 96, 21002 (2011).

[2] G. Antchev, et al., EPL 101, 21002 (2013).

[3] G. Antchev, et al., EPL 101, 21004 (2013).

[4] G. Antchev, et al., Phys. Rev. Lett. 111, 012001 (2013).

[5] P. Abreu, et al., Phys. Rev. Lett. 109, 062002 (2012).

[6] L. A. Fajardo et al. Phys. Rev. D 24, 46 (1981).

[7] U. Amaldi and K. R. Schubert, Nucl. Phys. B 166, 301 (1980).
[8] C. Augier et al. UA4/2 Collaboration. Phys. Lett. B 316, 448 (1993).

[9] N. A. Amos et al. Phys. Rev. Lett. 68, 2433 (1992).

[10] D. S. Ayres et al. Phys. Rev. D 15, 3105 (1977).

[11] C. W. Akerlof et al. Phys. Rev. D 14, 2864 (1976).

[12] G. Fidecaro et al. Phys. Lett. B 105, 309 (1981).

[13] R. Rubinstein et al. Phys. Rev. D 30, 1413 (1984).

[14] K. R. Schubert Vol. I/9a. Tables on nucleon-nucleon scattering. Springer-Verlag, Berlin. (1979)

[15] M. Bozzo et al. Phys. Lett. B 147, 385 (1984).

[16] M. Bozzo et al. Phys. Lett. B 155, 197 (1985).

[17] D. Bernard et al. Phys. Lett. B 171, 142 (1986).

[18] N. A. Amos et al. Phys. Lett. B 247, 127 (1990).

[19] R. J. Glauber, High energy physics and hadron structure. (Edited by S. Devons et al. Plenum, New York, 1970), p. 207.

[20] R. J. Glauber, J. Velasco, Phys. Lett. B. 147, 380 (1984).

[21] A. Sánchez-Hertz, thesis, Universidad Nacional Autónoma de México (2014).

[22] A. F. Martini, M. J. Menon, Phys. Rev. D 56, 4338 (1997).

[23] M. J. Menon, Can. J. Phys. 74, 594 (1996).

[24] J. Pérez Peraza, A. Sánchez Hertz, M. Alvarez Madrigal, A Gallegos Cruz, J. Velasco, A. Faus Golfe, New J. Phys. 7, 150 (2005).

[25] G. Matthiae, Rep. Prog. Phys. 57, 743 (1994).

[26] A. S. Carrol, et al., Phys. Rev. Lett. B. 61, 303 (1976).

[27] A. S. Carrol, et al., Phys. Rev. Lett. B. 80, 423 (1979).

[28] U. Amaldi, K. R. Schubert, Nucl. Phys. B. 166, 301 (1980).

[29] F. Abe, et al., Phys. Rev. D. 50, 5550 (1994).

[30] M. Bozzo, et al., Phys. Lett. B. 147, 392 (1984).

[31] G. J. Alner, et al., Z. Phys. C. 32, 153 (1986).

[32] C. Avila, et al., Phys. Lett. B. 445, 419 (1999).

[33] N. A. Amos, et al., Phys. Rev. Lett. 68, 2433 (1992).

[34] N. A. Amos, et al., Phys. Rev. Lett. 63, 2784 (1989).

[35] M. Honda, et al., Phys. Rev. Lett. 70, 525 (1993).

[36] R. M. Baltrusaitis, et al., Phys. Rev. Lett. 52, 1380 (1984). 